\title{
The evidence for expanded genetic testing for pediatric patients with cancer
}

\author{
Luke Maese ${ }^{1} \&$ Joshua D Schiffman*,1,2 \\ ${ }^{1}$ Department of Pediatrics, University of Utah, Salt Lake City, UT 84108, USA \\ ${ }^{2}$ Department of Oncological Sciences, Huntsman Cancer Institute, University of Utah, Salt Lake City, UT 84112, USA \\ * Author for correspondence: joshua.schiffman@hci.utah.edu
}

\begin{abstract}
"While these advances are a tremendous accomplishment, there are still many areas in which pediatric oncology has yet to realize the full potential of molecular medicine. Two of these areas, developmental therapeutics and cancer prevention techniques, harbor great opportunities for advancement that may be realized through an expansion of clinical cancer genetic testing."
\end{abstract}

First draft submitted: 13 September 2017; Accepted for publication: 1 November 2017; Published online: 12 January 2018 Keywords: genetics $\bullet$ genomics $\bullet$ hereditary cancer $\bullet$ next-generation sequencing $\bullet$ pediatric cancer $\bullet$ personalized
- precision $\bullet$ predisposition

The sequencing of the human genome had many consequences for the medical field, perhaps the greatest of which was spurring the development of personalized medicine. A vast expanse of data were generated, the likes of which had never been seen, opening up countless possibilities for patients. For the oncological community, the greatest impact of this data has been realized through the advent and evolution of molecular diagnostics and therapeutics giving rise to what is commonly known as 'precision oncology'. Precision oncology has evolved with technological advancements and currently encompasses the use of molecular data to inform decision-making regarding cancer prevention, diagnosis and treatment. Pediatric oncology has, in some ways, been at the forefront of integration of precision medicine into everyday practice. This is best personified in the care of the most common type of pediatric cancer acute lymphoblastic leukemia [1]. Molecular testing is now incorporated into diagnosis, risk assessment, response measurement and treatment of this once universally fatal disease, which now exhibits cure rates above $90 \%$ [2]. While these advances are a tremendous accomplishment, there are still many areas in which pediatric oncology has yet to realize the full potential of molecular medicine. Two of these areas, developmental therapeutics and cancer prevention techniques, harbor great opportunities for advancement that may be realized through an expansion of clinical cancer genetic testing.

\section{Precision medicine in pediatric oncology}

Like precision oncology, clinical cancer genetic testing has evolved encompassing a variety of modalities with the current era rooted in next-generation sequencing (NGS) techniques. A variety of different NGS assays are available: single gene testing, multigene panels, whole exome/whole genome sequencing and single nucleotide polymorphism microarrays; all of which have demonstrated the usefulness of genetic testing in the pediatric cancer population. There are two separate but equally important parts of cancer genetic testing in pediatric oncology, somatic and germline. Somatic tumor testing has received the bulk of attention, primarily for therapeutic implications, as the demonstrated effectiveness in identification of mutations, prescription of 'targeted therapy' and classification of known resistance mutations is well documented [3]. The bulk of NGS research in pediatric oncology has occurred in patients with relapsed/refractory malignancy making the utility of somatic tumor testing in every newly diagnosed patient unclear. Focused testing of this population has centered on their failure of conventional treatment. Research has sufficiently demonstrated the usefulness for testing in this high-risk population, as the largest most recently documented effort reported that nearly $70 \%$ of patients harbored druggable alterations in their tumors [4]. With the findings of this report and many others, somatic NGS testing for relapsed/refractory malignancy is becoming a mainstay of care for this population. An often overlooked value of somatic testing is the ability to enhance diagnostic

Future Medicine 
capabilities. While not commonplace, a change in diagnosis was recorded in over $4 \%$ of patients analyzed on a group of recent studies [5]. Given the psychological and physical implications of misdiagnosed childhood cancer, the impact of this cannot be understated. There is sufficient evidence to suggest any pediatric patient with relapsed, refractory and/or an unclear diagnosis should undergo somatic NGS. An additional and increasingly more frequently realized result of somatic testing is the ability of these assays to infer pathogenic germline mutations [6]. This along with the increased utilization of NGS has contributed to increased clinician awareness of hereditary cancer predisposition syndromes (CPS).

Germline testing, as opposed to somatic testing, is the gold standard for identification of CPS. Once thought to be a rare finding, incidence of germline hereditary cancer predisposition mutations in pediatrics is now estimated to be approximately $10 \%$ [7]. While this number is significant, greater than 1500 patients in the USA and 30,000 patients worldwide when considering current incidence of pediatric cancer, it is likely to increase given the broader utilization of NGS techniques, expansion of known identified cancer predisposition genes and the expanded availability of genetic-counseling services $[8,9]$. Additionally, these estimates gleaned from recently performed studies do not take into account potential interplay between somatic mutations and the germline. It has been hypothesized mutations in cancer predisposition genes that occur only in tumor tissue and also contribute to oncogenesis, emphasizing there is still much to be learned about cancer predisposition mutations [10]. Considering children meeting criteria for clinical cancer genetic-testing referrals are nearly $30 \%$ with actual referral rates of $6 \%$ the current prevalence of CPS are surely to rise [11].

\section{Benefits of expanded testing}

As increasing numbers of patients are tested, one may ask a question of utility, 'how does this information benefit patients'? There are tangible and intangible benefits to identification of hereditary cancer predisposition. If a person can be preemptively identified as at high risk for cancer, modern surveillance techniques allow for earlier identification of asymptomatic disease and in select situations improved outcomes [12,13]. Up until recently, there were few established guidelines for surveillance of pediatric patients with CPS, however, a recent workshop sponsored by the Pediatric Cancer Working Group of the American Association of Cancer Research consisting of an international expert panel of pediatric hematologist-oncologists, endocrinologists, radiologists, geneticists and genetic counselors convened in October 2016 to provide consensus-driven recommendations for early tumor screening for pediatric patients with CPS [14]. This workshop produced 17 publications including guidelines for genetic referral, testing and surveillance encompassing the 50 most common syndromes encountered in the pediatric age group. While much of the guidelines are primarily based on expert opinion, due to the novelty and lack of research in this field, they provide an outline for clinicians on how to manage CPS [15]. The ability to prescribe an intervention for a CPS is one of many advantages realized through early identification. Alerting the immediate and extended family to a previously unknown cancer risk enabling a pursuit of counseling and testing expands the scope of benefits. Additionally, parental perception of the value of germline testing extends beyond clinical management including the psychological and pragmatic value of the information [16]. This value is extended outside an individual family to the entire population as it has contributed to a broader understanding of cancer biology within and beyond the genome [17].

Current protocol for CPS referral/testing varies according to institutional practice often reflecting available resources. There are several resources, in addition to the American Association of Cancer Research Workshop, aiding providers in deciding whom a CPS should be considered [18,19]. A major component of these tools is the ability to record a complete and accurate family history which for numerous reasons remain a weakness of oncology providers [20,21]. This combined with the possibility of de novo variants, parental germline mosaicism, penetrance variability, recessive inheritance, small families and an incomplete understanding of hereditary cancer predisposition limits the current recommendations and gives credence to an expansion of CPS screening [22].

\section{Challenges of expanded testing}

There are challenges with expanded usage of clinical cancer genetic testing in pediatric cancer patients. In the current fiscal climate, any expansion of testing should consider financial implications. Along with the precipitous drop in cost of NGS techniques [23], many commercial testing companies offer excellent financial assistance programs in some cases performing it free of charge. Cost has become less of a barrier to testing and storage/interpretation of data are quickly becoming the rate-limiting step [24]. Cost-effectiveness of surveillance programs is an understudied area of precision oncology, however, for $\mathrm{Li}$-Fraumeni syndrome surveillance was demonstrated in one analysis to 
be cost-effective [TAK C et al., Unpublished Data]. Ethical and psychological concerns are an ever-present topic whenever the subject of genetic testing arises. Pediatric clinical cancer genetic testing growth needs to include a broadening availability for genetic counseling services as a lack of informed counseling prior to testing is associated with adverse early tumor surveillance and psychosocial outcomes [25]. Although there is increased availability of commercial NGS, it is essential, whenever possible, for pediatric clinical cancer genetic testing to take place in the setting of a clinical trial. This is particularly important as more/larger tissue banks, improved genomic classifications and refinement of current estimated rates of hereditary cancer predisposition are required to better inform the community on pediatric clinical cancer genomics $[7,26]$.

\section{Conclusion}

Given the current available evidence, somatic tumor testing should be pursued in all patients with relapsed, refractory and/or diagnostic uncertainty. Additionally, at the current estimates of CPS combined with the likelihood that these are underestimated numbers, the lack of recognition by primary providers of potential CPS, the currently available guidelines for management of CPS and the decreasing cost of sequencing an expansion of CPS testing is warranted. However, it is vital to consider resources for genetic counseling and testing prior to germline genetic testing and enrollment of patients on clinical trials should be prioritized.

\section{Acknowledgements}

JD Schiffman holds an Edward B Clark, MD Chair in Pediatric Research, and is supported through the Primary Children's Hospital $(\mathrm{PCH})$ Pediatric Cancer Program funded by the Intermountain Healthcare Foundation and the PCH Foundation, the Li-Fraumeni Syndrome Association (LFSA), and Soccer For Hope Foundation.

\section{Financial \& competing interests disclosure}

JD Schiffman is co-founder and shareholder of ItRunsInMyFamily.com, an online family history collection tool. The authors have no other relevant affiliations or financial involvement with any organization or entity with a financial interest in or financial conflict with the subject matter or materials discussed in the manuscript apart from those disclosed.

No writing assistance was utilized in the production of this manuscript.

\section{References}

1. Tasian SK, Loh ML, Hunger SP. Childhood acute lymphoblastic leukemia: integrating genomics into therapy. Cancer 121(20), 3577-3590 (2015).

2. Pui C-H, Yang JJ, Hunger SP et al. Childhood acute lymphoblastic leukemia: progress through collaboration. J. Clin. Oncol. 33(27), 2938-2948 (2015).

3. Gagan J, Van Allen EM. Next-generation sequencing to guide cancer therapy. Genome Med. 7(1), 80 (2015).

4. Worst BC, Pfaff E, Tilburg CMV et al. The INFORM personalized medicine study for high-risk pediatric cancer patients. J. Clin. Oncol. 35(Suppl. 15), 10509 (2017).

5. Mody RJ, Prensner JR, Everett J, Parsons DW, Chinnaiyan AM. Precision medicine in pediatric oncology: lessons learned and next steps. Pediatr. Blood Cancer 64(3), doi:10.1002/pbc.26288 (2017).

6. Raymond VM, Gray SW, Roychowdhury S et al. Germline findings in tumor-only sequencing: points to consider for clinicians and laboratories. J. Natl Cancer Inst. 108(4), pii: djv351 (2016).

7. Zhang J, Walsh MF, Wu G et al. Germline mutations in predisposition genes in pediatric cancer. N. Engl. J. Med. 373(24), 2336-2346 (2015).

8. Schiffman JD. Hereditary cancer syndromes: if you look, you will find them. Pediatr. Blood Cancer 58(1), 5-6 (2012).

9. Steliarova-Foucher E, Colombet M, Ries LAG et al. International incidence of childhood cancer, volume III. International Agency for Research on Cancer. http://iicc.iarc.fr/results/

10. Rahman N. Realizing the promise of cancer predisposition genes. Nature 505(7483), 302-308 (2014).

11. Knapke S, Nagarajan R, Correll J, Kent D, Burns K. Hereditary cancer risk assessment in a pediatric oncology follow-up clinic. Pediatr. Blood Cancer 58(1), 85-89 (2012).

12. Villani A, Shore A, Wasserman JD et al. Biochemical and imaging surveillance in germline TP53 mutation carriers with Li-Fraumeni syndrome: 11 follow-up of a prospective observational study. Lancet Oncol. 17(9), 1295-1305 (2016).

13. Malkin D, Nichols KE, Zelley K, Schiffman JD. Predisposition to pediatric and hematologic cancers: a moving target. Am. Soc. Clin. Oncol. Educ. Book 2014, e44-e55 (2014). 
14. Brodeur GM, Nichols KE, Plon SE, Schiffman JD, Malkin D. Pediatric cancer predisposition and surveillance: an overview, and a tribute to Alfred G Knudson Jr. Clin. Cancer Res. 23(11), e1-e5 (2017).

15. Clinical Cancer Research. http://clincancerres.aacrjournals.org/pediatricseries

16. Malek J, Slashinski MJ, Robinson JO et al. Parental perspectives on whole-exome sequencing in pediatric cancer: a typology of perceived utility. JCO Precis. Oncol. doi:10.1200/po.17.00010 (2017).

17. Samuel N, Villani A, Fernandez CV, Malkin D. Management of familial cancer: sequencing, surveillance and society. Nat. Rev. Clin. Oncol. 11(12), 723-731 (2014).

18. Jongmans MC, Loeffen JL, Waanders $\mathrm{E}$ et al. Recognition of genetic predisposition in pediatric cancer patients: an easy-to-use selection tool. Eur. J. Med. Genet. 59(3), 116-125 (2016).

19. Ripperger T, Bielack SS, Borkhardt A et al. Childhood cancer predisposition syndromes - a concise review and recommendations by the Cancer Predisposition Working Group of the Society for Pediatric Oncology and Hematology. Am. J. Med. Genet. A 173(4), 1017-1037 (2017).

20. Welch BM, Dere W, Schiffman JD. Family health history: the case for better tools. JAMA 313(17), 1711-1712 (2015).

21. Wood ME, Kadlubek P, Pham TH et al. Quality of cancer family history and referral for genetic counseling and testing among oncology practices: a pilot test of quality measures as part of the American Society of Clinical Oncology Quality Oncology Practice Initiative. J. Clin. Oncol. 32(8), 824-829 (2014).

22. Druker H, Zelley K, Mcgee RB et al. Genetic counselor recommendations for cancer predisposition evaluation and surveillance in the pediatric oncology patient. Clin. Cancer Res. 23(13), e91-e97 (2017).

23. Wetterstrand KA. DNA sequencing costs: data from the NHGRI Genome Sequencing Program (GSP). www.genome.gov/sequencingcost

24. Muir P, Li S, Lou S et al. The real cost of sequencing: scaling computation to keep pace with data generation. Genome Biol. 17(1), 53 (2016).

25. Bensend TA, Veach PM, Niendorf KB. What's the harm? Genetic counselor perceptions of adverse effects of genetics service provision by non-genetics professionals. J. Genet. Couns. 23(1), 48-63 (2014).

26. Janeway KA, Place AE, Kieran MW, Harris MH. Future of clinical genomics in pediatric oncology. J. Clin. Oncol. 31(15), 1893-1903 (2013). 